\title{
"Sollten unsere Vorfahren Menschen gewesen sein wie diese?" Versuch einer postkolonialen Lektüre von Eberhard Hilschers Darwin-Erzählung
}

\begin{abstract}
"Should our Antedates Have Been People Like These?“

A Try of Postcolonial Reading of the Story about Darwin by Eberhard Hilscher

The following paper presents a postcolonial reading (combined with the ideology-critical approach) of the little-known story about Darwin by the East German writer Eberhard Hilscher. The analysis focuses on the perception of the foreign and the own by the figures of the story and it also refers to the overlapping of the terms race and class. The considerations lead to the conclusion that in the story the foreign is constructed preferably with the help of the opposition culture (the own) and nature (the foreign), and that hierarchical and dichotomous thinking characterizes also Darwin, even if in his case one can see certain ambivalences.
\end{abstract}

Keywords: postcolonial reading, Eberhard Hilscher, Darwin, GDR, the foreign, the own, ideology.

Schlüsselwörter: postkoloniale Lektüre, Eberhard Hilscher, Darwin, DDR, das Fremde, das Eigene, Ideologie.

Wer sich heute auf das Terrain der postkolonialen Theorien wagt, müsse sich seiner schwierigen Ausgangslage bewusst sein und ein hohes Maß an Selbstreflexivität mitbringen, so Ida Kerner in ihrer einschlägigen Monografie Postkoloniale Theorien zur Einführung (2012). Diese Behauptung trifft ohne Zweifel zu: Die postkolonialen Theorien, wie sie vorzugsweise von der sog. Trinität, d.h. von Edward Said, Homi Bhabha und Gayatri Spivak profiliert wurden, bilden ein derart heterogenes und dynamisches Theoriefeld ${ }^{1}$, dass man Gefahr läuft, in dem Dic-

1 Vgl. I. Kerner, Postkoloniale Theorien zur Einführung, Hamburg 2012, S. 18-19. 
kicht des bereits Beschriebenen die Orientierung zu verlieren, zum unbewussten Nachahmer etablierter Denkmuster zu werden oder innerhalb voreingenommener Sichtweisen zu erstarren. Und dies wäre gerade das, was die postkolonialen Theoretiker zu vermeiden versuchen: die Schließung und eine eindeutige Bestimmung bzw. Schubladisierung, denen ja häufig Hierarchien und Dichotomien zugrunde liegen, welche, so zumindest die stillschweigende Annahme, positionierendes und kategorisierendes Denken erzwingen. Doch ohne jene gedanklichen Orientierungssäulen, wie sie zwangsläufig aufgebaut werden müssen, um überhaupt einen interpretatorischen Zugriff vornehmen zu können, scheint mir jedweder Ansatz einer postkolonialen Lektüre, wie ich sie im Folgenden versuchen will, im Keim ersticken zu drohen. Jene Kategorisierungen scheinen mir daher unumgänglich zu sein: sie wollen jedoch nicht im normativen, sondern im deskriptiven Sinne verstanden werden.

Um möglichen Missverständnissen vorzubeugen, soll daher bereits im Vorfeld gesagt werden, dass es in den nachfolgenden Ausführungen weniger um die postkoloniale Theorie bzw. postkoloniale Theorien, sondern vielmehr um deren praktische Anwendung, d.h. eine an den postkolonialen Theorien orientierte Lektüre gehen wird. Zentrale Begriffe und Fragestellungen sollen zwar in diese Lesart Eingang finden, sie werden jedoch ihre Richtung nicht vorbestimmen. Mit der postkolonialen Lesart will ich folglich eine solche Lektüre verstehen, die sich auf die literarischen Konstruktionen des Eigenen und des Fremden sowie die Problematik der Fremdheitszuschreibung und -wahrnehmung, die in der Regel mit der Herausbildung hierarchischer Oppositionen zwischen der kulturellen Mehrheit und der Minderheit einhergeht ${ }^{2}$, fokussiert, die aber nicht nur die inhaltlich-symbolische Aufladung, sondern auch formale Besonderheiten solcher Konstruktionen berücksichtigt. In Anlehnung an die Postulate Neumanns ${ }^{3}$ soll diesen Fragestellungen mit dem Instrumentarium der strukturalen Analyse begegnet werden, welche sich vorzugsweise auf die Figuren als textimmanente Größen und Träger eines für das gewählte Werk spezifischen Diskurses über das Eigene und Fremde konzentrieren wird. Die von Lubrich aufgeworfene Frage: „In welcher Weise dient eine literarische Konstitution des 'Fremden' seiner ideologischen Abwehr, Bewältigung oder Kontrolle?" ${ }^{\star 4}$ soll dabei nicht weniger Aufmerksamkeit erfahren.

Die in der ehemaligen DDR fünfmal aufgelegte Darwin-Erzählung von Eberhard Hilscher (1927-2005) wurde zum ersten Mal unter dem Titel Feuerland ahoi! im Paul List Verlag publiziert (1961). Die Erzählung gewann ihren ursprünglichen (vom Verlag gegen den Willen des Autors veränderten) Titel Mister Darwin macht eine Entdeckung - erst infolge der Zusammenarbeit Hil-

2 Vgl. B. Neumann, Methoden postkolonialer Literaturkritik und anderer ideologiekritischer Ansätze [in:] Methoden der literatur- und kulturwissenschaftlichen Textanalyse, Hrsg. V. Nünning, A. Nünning, Stuttgart-Weimar 2010, S. 271-292, hier S. 271.

3 Ibid., S. 274.

4 O. Lubrich, Welche Rolle spielt der literarische Text im postkolonialen Diskurs? [in:] Archiv für das Studium der neueren Sprachen und Literaturen, 242. Band, 157, Hrsg. H. Brunner, C. Jansohn, M. Lentzen, D. Mehl, 2005, S. 16-39, hier S. 17. 
schers mit dem Verlag der Nation, der für die Neuauflagen sorgte. Abgesehen von der kleineren Erzählung über Galileo Galilei, welche 1956 u.d.T. Der Inquisition verfallen zum ersten Mal in der ,Wochenpost" erschienen ist, kann die DarwinErzählung als das eigentliche Buchdebüt des studierten Germanisten Hilscher angesehen werden. ${ }^{5}$ Bis heute ist das Buch aber wenig bekannt - in die deutsche Literaturgeschichte schrieb sich der Autor vor allem dank seinen Romanen: Der Morgenstern oder die vier Verwandlungen eines Mannes, Walther von der Vogelweide genannt (1976) und Die Weltzeituhr (1983) ein, seinem opus magnum, das 2017 im Mitteldeutschen Verlag als Ausgabe letzter Hand neu aufgelegt wurde. Einen Namen hat sich aber Hilscher auch als Autor von viel beachteten Monographien und literaturwissenschaftlichen Essays über Arnold Zweig, Thomas Mann und Gerhart Hauptmann gemacht.

Die Darwin-Erzählung wurde bisher aus dem Blickwinkel der biografischen Treue und als ein Beispiel einer gelungenen Kombination wissenschaftlichen und unterhaltenden Erzählens gelesen. ${ }^{6}$ Verwiesen wurde ebenfalls auf die ihr innewohnende pädagogische Tendenz (so vor allem die DDR-Kritiker) sowie die Fabulierfreudigkeit des Autors. ${ }^{7}$ Kaum beachtet wurde dagegen die Tatsache, dass Hilscher hier in erster Linie die Begegnung eines Europäers - bzw. des „weißen“ Mannes - mit einer ihm fremden Kultur thematisiert und einen eigenen Fremdheitsdiskurs entwickelt, der nicht nur am Beispiel seines reisenden Helden ausgetragen wird, sondern auch seine Konturen aus der Gegenüberstellung zweier Welten - der „Ersten Welt“ (der kolonialen Macht) und der „Dritten Welt“ (der Kolonisierten) - gewinnt. Hilscher lässt nämlich seine Erzählung in zwei Handlungsstränge zerfallen: Während der erste Strang Darwin und seiner Reise gewidmet ist, gewährt der zweite Strang Einblicke in die politisch-soziale Realität im England in der ersten Hälfte des 19. Jahrhunderts, wobei das Augenmerk insbesondere auf die Kinderarbeit, die Revolte der Arbeiter und die Wahlkampagne der Whigs (der Liberalen) und der Torys (der Konservativen) und die damit verbundene Debatte um die Sklaverei gerichtet wird. Der Autor spielt die Wirklichkeit Südamerikas gegen die europäische Realität aus und entwickelt so einen (post) kolonialen Diskurs, der sich an den Fragen der Rasse und der Klasse entzündet.

Dabei ist zu beachten, dass für diesen Diskurs nicht nur die Person des Autors ausschlaggebend ist: Die Erzählung ist ja in dem „,besseren Deutschland“, wie sich die DDR selbst zu nennen pflegte, entstanden und publiziert wurde sie ebenfalls in einem ostdeutschen Verlag, dessen Lektor in seinem Gutachten den Autor für die angemessene Darstellung des ,soziale[n] Aspekt[s]“68 lobte: „der Autor

5 Vgl. N. Nowara-Matusik, Oblicza artystów, Kraków 2016, S. 47.

6 Vgl. W. Meissner, Eberhard Hilscher: Mister Darwin macht eine Entdeckung, „Neue deutsche Hefte“, Berlin (West) 1981, Nr. 170, Heft 2, S. 380-381.

7 Vgl. K. Walther, Biographie in Klein- und Großformat, „Neue Deutsche Literatur“ 1963, Heft 12, S. 159-160; Hawe (Harald Wessel), Weltreise zur Erkenntnis, „Neues Deutschland“, 21.04.1962, S. 8.

8 Lektoratsgutachten, Eberhard Hilscher, Mister Darwin macht eine Entdeckung (Arbeitstitel) (163 S.) http://www.argus.bstu.bundesarchiv.de/dr1_druck/index.htm?search=Hilscher\%20 Darwin\&Kontext $\mathrm{Fb}=$ KontextFb\&searchType $=$ all\&searchVolumes $=$ all\&highlight $=$ true $\&$ vid $=$ dr1_druck\&kid=5f9b7f19-e705-47b7-a5a4-005e6834e853\&uid=4e6fd242-103d-4c3e-a255cdbd08e368dd\&searchPos=1 (Zugriff: 08.07.2017). Aus den im bundesdeutschen Internet-Archiv 
zeigt, wie Darwin bei seinen Reisen im Ausland neben seinem naturwissenschaftlichen Hauptanliegen seinen stets wachen, kritischen Blick für gesellschaftliche Mißstände schärft und ein soziales Gewissen entwickelt, das die progressiven Züge seines Weltbildes auch nach dieser Seite hin abrundet" ${ }^{\text {.9 }}$ Daher muss die Analyse dieser Erzählung, die ,einen erschütternden Einblick in die Lage der arbeitenden Klassen im frühkapitalistischen England" ${ }^{\text {"10 }}$ gibt, auch ideologiekritisch ausgerichtet werden, zumal die DDR im Artikel 6 der Verfassung vom 6. April 1968 (in der Fassung vom 7. Oktober 1974) stolz verkündete, dass sie „die Staaten und Völker, die gegen den Imperialismus und sein Kolonialregime, für nationale Freiheit und Unabhängigkeit kämpfen, in ihrem Ringen um gesellschaftlichen Fortschritt"11 unterstützt. Antikolonialismus, Antirassismus und Solidarität mit den Unterdrückten als Verfassungsgrundsätze finden Eingang in den öffentlichen politischen Diskurs, wovon Artikelüberschriften wie DDR verurteilt Kolonialismus und Rassenpolitik ${ }^{12}$ oder Schlu $\beta$ mit dem Kolonialismus $!^{13}$ eindringlich Zeugnis ablegen. Wenn man etwas generalisierend feststellt, dass die in der DDR publizierte Literatur unter dem Druck ideologisch konformer Bilder produziert wurde, so liegt hier die Vermutung nahe, dass auch Hilschers Buch sich in jenes ideologische Paradigma des Antikolonialismus einschreiben musste. Mit anderen Worten: wollte ein Autor, dass die Zensurbehörden sein Buch zum Druck zulassen, so musste er nolens volens gewisse Kompromisse eingehen oder Geständnisse akzeptieren. ${ }^{14}$ Doch sollen hier keine voreiligen Schlüsse gezogen werden.

Der Kolonisierungsgedanke erscheint gleich am Anfang der Novelle, als Darwins Vater die Feuerländer als Barbaren imaginiert, welche man zu Gott bekehren sollte. ${ }^{15}$ Damit beschwört er ein weit verbreitetes Klischee, das das abendländische Selbstverständnis bis heute bestimmt, nämlich das Oppositionspaar „Zivilisation“ versus „Barbarei“. Der Held will unmissverständlich christianisieren, sprich: also auch erobern und so seinen eigenen Willen, dem sich die „Wilden“ unterzuordnen haben, durchsetzen. Damit vertritt er eine Weltsicht, in die sich auch andere Figuren der Novelle einreihen lassen, nämlich die eines typischen Kolonialherren, des Vertreters der imperialen Macht, welcher aus der überlegenen

vorhandenen Akten des Verlags ergibt sich, dass Hilscher mehrere Aspekte seiner Erzählung unter dem Druck des Verlags verändern musste. Der Autor hat u.a. den Realitätsbezug der Erzählung stärker akzentuiert, Darwins Forscherdrang hervorgehoben und die erotischen Szenen, an denen wahrscheinlich der Gutachter Anstoß nahm, etwas ,gemäßigt“. Diese Veränderungen lassen darauf schließen, dass der Verlag vor allem darum bemüht war, die Erzählung etwas stärker den Prinzipien des sozialistischen Realismus anzupassen und Darwins Weltanschauung im Einklang mit dem Materialismus darzustellen.

9 Ibid.

10 Ibid.

11 http://www.documentarchiv.de/ddr/verfddr.html (Zugriff: 08.07.2017).

12 (ADN), DDR verurteilt Kolonialismus und Rassenpolitik, „Neues Deutschland“, 29.01.1965, S. 7.

13 (NZ/ADN), Schluß mit dem Kolonialismus!, „Neue Zeit“", 8.04.1955, S. 2.

14 Dies ist selbstverständlich eine Vereinfachung - über das Funktionieren der Zensur in der DDR und ihre Literaturpolitik siehe mehr z.B. in: Cenzura w Niemczech $w$ XX wieku. Studia, analizy, dokumenty, wybór i opracowanie Cz. Karolak, Poznań 2000.

15 E. Hilscher, Mister Darwin macht eine Entdeckung, Erzählung-Berlin 1989, S. 11. Alle weiteren Zitate beziehen sich auf diese Textausgabe. 
Position eines Europäers spricht und keinerlei Skrupel oder Bedenken im Umgang mit dem durch ihn abgewerteten Fremden zeigt.

In diese Figurengruppe lässt sich auch Kapitän Robert Fitz-Roy einordnen, mit welchem Darwin auf seinem Schiff „Beagle“ um die Welt reist. Ähnlich wie Darwins Vater glaubt er, dass die Feuerländer ,,hinterwäldlerisch“ leben und dass man sie zu ,anständigen Christenmenschen“ (S. 21) machen solle. Als überzeugter Tory ist er deswegen empört, dass die Whigs eine Kampagne gegen die Sklaverei angefangen haben und seine Versklavung dreier Indianer, ,seine hochherzige Zivilisationstat an den drei Feuerländern“ (S. 23), heftig kritisieren, wo er doch ,die drei aus dem Stande urtümlichster Unschuld in die großbritannische Hochzivilisation versetzt" (S. 23) hatte. Der englische Kapitän will offenbar die Indigenen „,kultivieren“, wobei er seine Pläne in der zivilisatorischen Überlegenheit des englischen Empires begründet sieht. Damit erfolgt eine kulturelle Rechtfertigung des Kolonialismus, wie sie für die englische Literatur des 19. Jahrhunderts typisch ist. ${ }^{16}$

Am Beispiel dieser Figurengruppe diskutiert Hilscher jedoch keine eigentümlichen Sichtweisen, sondern solche, die sich mentalitätsgeschichtlich erklären lassen: Die hier erwähnten Figuren sind zwar individuell angelegt, sie stehen aber zugleich emblematisch für typische englische Kolonisten (auch wenn sie im wortwörtlichen Sinne keine Eroberer sind), welche die britische civilizing mission erfüllen wollen.

Die zweite Figurengruppe bilden Gestalten, die ebenfalls Kolonisierungsabsichten hegen, mit dem Unterschied, dass jene nicht auf das Fremde, sondern auf das Eigene gerichtet sind. Ein herausragendes Beispiel ist hier Professor John Steffen Henslow, der Lehrer von Charles Darwin, welcher sich der Meinung eines Journalisten anschließt, der ,das englische Volk mit unmündigen Kindern“" (S. 13-14) vergleicht: Mit dieser Aussage degradiert er die „einfachen Leute“ (S. 14) zu willenlosen und quasi voraufklärerischen Wesen, sich selbst in der Rolle des aufgeklärten Herrschers positionierend. In den England-Kapiteln liest man ebenfalls von einem Fabrikanten, welcher den Typus des unmenschlichen, schlauen und nur auf seinen Gewinn erpichten Kapitalisten und Ausbeuters darstellt. Was augenfällig ist, ist, dass diese Figur ihren Pendant in den Amerika-Kapiteln findet: Darin begegnet man einem gewissen Don Manuel, einem im Überfluss lebenden Gutsbesitzer, der hungrige „Negerkinder“ (S. 76) von der Türe wegjagt. Dass der argentinische Gutsbesitzer das Spiegelbild des englischen Fabrikanten ist, scheint offensichtlich zu sein.

Die parallele Gegenüberstellung von Figuren in den England- und Amerika-Kapiteln ist ein erzähltechnisches Verfahren, mit dem Hilscher die Diskurse über die Rasse und die Klasse sich überlappen und gegenseitig durchdringen lässt. Man könnte sogar meinen, der Autor würde einen gemeinsamen Erfahrungsraum erschaffen, indem er die distinktiven Merkmale der englischen und der südamerikanischen Realität der unterdrückten indigenen Völker und Arbeiter in eine

16 Vgl. M. Varela do Mar Castro, N. Nikita, Postkoloniale Theorie. Eine kritische Einführung. Bielefeld 2005, S. 156. 
auffallende Nähe rücken lässt: Allen voran nimmt er die sozialen, d.h. klassenbedingten, Ungerechtigkeiten in den Blick. Damit erstellt er Parallelen und zieht mentale Verbindungslinien, die als scheinbar kontrastierende Einblenden ${ }^{17}$ in die Handlung montiert werden. Somit wird der Leser aufgefordert, die Zusammenhänge - und nicht die Differenzen - wahrzunehmen: zwischen der Ausbeutung der Arbeiter in dem frühkapitalistischen England und der Unterdrückung der versklavten indigenen Völker in den englischen Kolonien. Der Gedanke, den Hilscher hier mit hoher Wahrscheinlichkeit sichtbar machen will, lässt sich wie folgt zusammenfassen: Der Kolonialismus, ähnlich wie der Kapitalismus, gründet sich auf der totalen Ausbeutung der Schwachen und Entmündigten. Man könnte sogar den Eindruck gewinnen, dass der Autor die Suggestion vermitteln will, ähnlich wie Franz Fanon in Die Verdammten dieser Erde (1961), dass es einen engen Zusammenhang zwischen den sich weltweit abspielenden klassen- und rassebedingten Konflikten gebe. So betrachtet beispielsweise Darwin - ganz im Sinne des sozialistischen Bewusstseins - die mancherorts in Südamerika ausbrechenden Unruhen als gerechten Kampf der Eroberten gegen die Eroberer für die ihnen gewaltsam entrissene Freiheit (vgl. S. 126), wobei der Kampf der Indianer an den englischen „Swing“ denken lässt, wie Hilscher die Revolte der englischen Arbeiter bezeichnet. Sowohl die südamerikanischen Indianer als auch die englischen Arbeiter werden als Sklaven und Opfer eines ungerechten Systems dargestellt, wobei ihre Sklavenarbeit der Aufrechterhaltung jenes Systems dient: des Kapitalismus in England und des Kolonialismus in Südamerika.

Eine besondere Stellung innerhalb des Figurenensembles kommt selbstverständlich Charles Darwin zu. Seine Wahrnehmung des Fremden wird aber von Ambivalenzen und Widersprüchen getragen, welche auf verschiedenen Etappen seiner Reise zum Vorschein kommen.

Die erste Begegnung Darwins mit der südamerikanischen Fremde ist signifikanterweise die Begegnung mit der fremden Natur: Als der Forscher den exotischen Urwald betritt, ist er von den auf ihn zukommenden Geräuschen überwältigt und beunruhigt:

Das Vorwärtskommen gestaltete sich immer schwieriger. Die Bäume drängten sich dick verfilzt zusammen und versperrten den Weg durch Lianenvorhänge. Schwüle Dünste nahmen dem Europäer fast die Besinnung, das Hemd klebte an ihm: hinter dem undurchdringlichen grünen Wald gellte immer wieder das unerträgliche Iba-Oba-Uba-Geschrei. Der helle Anfangslaut klang wie Gelächter. Es war, als lache jemand die Männer aus, die sich abmühten, im Dickicht vorzudringen (S. 61-62).

17 Cem Sengül sieht in den eingeblendeten Kapiteln die Tendenz, ,das unkonventionelle Forscherleben mit dem Alltag der feinen britischen Gesellschaft zu kontrastieren“ (C. Sengül, Wissenschaft als Abenteuer in Eberhard Hilschers Darwin-Erzählung [in:] Eberhard Hilscher (19272005). Schriftsteller und Forscher der deutschen Literatur. Pisarz i badacz literatury niemieckiej, Hrsg. G.B. Szewczyk, Świebodzin-Katowice 2010, S. 34-42, hier S. 36). Diese Behauptung darf man nicht unwidersprochen stehen lassen: Der Kontrast zeichnet sich zwar deutlich ab, wenn es um die Haupthandlung geht, die scheinbar zweitrangigen Handlungsstränge der Darwin-Reise finden aber ihre Entsprechungen in den England-Kapiteln, so dass man eher von einem spiegelbildlichen, also einem auf die Herstellung von gedanklichen Analogien - und nicht von Kontrasten - zielenden Effekt, sprechen sollte. 
Kennzeichnenderweise wird Darwin wieder Herr über sein erschüttertes Ich, als er ein Äffchen erschießt: Von diesem Moment an jagt ihm auch das unheimliche Geschrei der Tiere keine Angst mehr ein. Der quasi natürliche Gewaltakt - die Erschießung eines jungen und unschuldigen Affen - lässt sich dabei wie ein symbolischer Akt der Eroberung des Fremden lesen, welcher die Dominanz der Europäer legitimiert - das Fremde hat von nun an auf den kommenden Herren zu hören. Auch wenn sich diese Tat Darwins als eine logische Konsequenz seiner Forschernatur erweist - er braucht ja das Äffchen als Untersuchungsgegenstand - so schimmert hier der äußerst beunruhigende Gedanke durch, dass das Fremde, soweit es sich nicht dem Eigenen erschließt und unterwürfig zeigt, einfach in eine andere Welt hinübergeschafft werden kann. ${ }^{18}$

Als jedoch Darwin dann endlich dem Fremden in Gestalt der Afroamerikane begegnet, wird seine Perzeption der Fremde nicht von Emotionen, sondern von der Ratio getragen. Sichtbar ist auch seine Sympathie für die ausgebeuteten Menschen:

Seit ich die Zustände hier in Bahia gesehen habe, ist es mir unmöglich, den Negern mein Mitgefühl zu versagen. Sie werden mir zugeben müssen, daß es unter ihnen prächtige Menschen gibt, Leute mit einem fröhlichen, ehrlichen Wesen, die Besseres verdient hätten, als von ihren weißen Herrschaften wie Vieh behandelt zu werden (S. 68).

Darwin kritisiert ohne Zweifel die imperiale und koloniale Politik Englands, wobei er den Fremden - das soll hier ausdrücklich akzentuiert werden - den Status der Menschen, welchen unverdientes Unrecht geschieht, nicht abspricht. Er weiß auch sogleich ihre Lage zu verbessern: „Hätten die Farbigen dieselben Bildungsmöglichkeiten wie wir, so würden sie bestimmt Beträchtliches leisten. Ich muß daher fragen, ob es nicht unseres Vaterlandes würdiger wäre, den Kolonialvölkern die Freiheit zu schenken und die Sklaverei völlig abzuschaffen“" (S. 68). Darwins Aussage liest sich wie eine Gegenrede zur imperialen Weltsicht: Indem er die ausbeuterischen und erniedrigenden Praktiken der westlichen Kultur blossstellt, stellt er sein ideologisches Statement auf, das wie eine treuherzige Deklaration eines DDR-Bürgers anmutet: Als Verfechter des sozialistischen Fortschritts, des Kampfes gegen den Kolonialismus und Kapitalismus wird Darwin in dieser Passage zum Aushängeschild einer sozialistischen Gesinnung. Dieses ideologische Konstrukt erweist sich aber im Laufe der Lektüre als äußerst instabil und sogar ambivalent - denn trotz seiner deklarierten Fortschrittlichkeit zeigen sich in seiner Perzeption des Fremden viele für den kolonialen - sprich also auch: reaktionären - Diskurs typische Klischees und Vorstellungsbilder. Dies lässt sich zum Beispiel dem folgenden Zitat entnehmen:

Charles Darwin wandte den Feuerländern während des ganzen Aufenthaltes seine höchste Aufmerksamkeit zu. (...) Zunächst einmal hatte er nicht die geringste Erfahrung im

18 Dies wird in einer anderen Episode, die mit der Äffchen-Szene scheinbar nichts zu tun hat, angedeutet: In einem beiläufigen Kommentar des Erzählers erfährt man nämlich, dass viele englische Arbeiter, die in England für die Unruhen gesorgt haben, verschwunden sind oder nach Australien befördert wurden. Parallelen liegen somit auf der Hand: die Befindlichkeit der kolonialen Völker soll in Analogie zu der Lage der entmündigten Arbeiter gelesen werden. 
Umgang mit so urwüchsigen Naturkindern (...). Er fand ihr Dasein primitiv (...). Zur Nahrung dienten ihnen Miesmuscheln, Beeren, Tran und fauliger Walspeck, weshalb Darwin auch eine ungeheuerliche Legende für glaubhaft hielt, derzufolge die Feuerländer in Hungerszeiten ihre alten Weiber töteten und auffraßen. (...) In seinem Tagebuch sprach Darwin immer wieder von seiner Erschütterung über diese „Wildheit“, „Primitivität“ und äffische „Nachahmungssucht". Gleichzeitig aber wurde er sich bewußt, wie verschieden die Menschen auf der Erde doch geartet waren. Es gab offenbar stark voneinander abweichende Entwicklungsstufen, und mit einer gewissen Beklommenheit stellte der junge Forscher am Ende die Frage: Sollten unsere Vorfahren Menschen gewesen sein wie diese? (S. 113)

Der Vergleich mit einer entsprechenden Stelle aus dem wohl bekanntesten Text, welcher in der einschlägigen Literatur als geradezu das Paradebeispiel der kolonialen Vereinahmung der Fremde immer wieder angeführt wird - Joseph Conrads Das Herz der Finsternis (1902) - mag in diesem Kontext erhellend sein:

Die Erde schien unirdisch. Wir mögen es gewohnt sein, die hingestreckte Gestalt eines besiegten Untiers zu betrachten, hier sah man aber einem Ding ins Auge, das ungeheuerlich und frei war. Es war unirdisch, und die Menschen waren (...) Nein, sie waren nicht unmenschlich. Und seht ihr, das war das Schlimmste dabei - dieser Verdacht, daß sie eben nicht unmenschlich waren. Es überkam einen ganz langsam. Sie heulten und sprangen und drehten sich und schnitten furchtbare Gesichter; was einen aber peinigte, war der Gedanke an ihre Menschlichkeit, gleich der eigenen, der Gedanke, daß man mit diesem wilden und verzweifelten Aufruhr entfernt verwandt war ${ }^{19}$.

Der Gedanke an die Verwandtschaft des weißen Mannes mit den Wilden, der Conrads Erzähler sichtlich verstört, ist auch bei Hilscher erschreckend gegenwärtig. Wie so typisch für den kolonialen Diskurs, liegt auch der Weltanschauung Darwins die Vorstellung zugrunde, dass die westliche Kultur ausschließlich für die Menschlichkeit und somit für den Humanismus steht, während das Fremde als das Unmenschliche per se präfiguriert wird. Ein verblüffender Rücktritt, wenn man bedenkt, dass der Held in der vorherigen Episode den Fremden nicht ihre Menschlichkeit abgesprochen hat. Diesmal aber befremdet er den Leser, indem er den Feuerländern einen kategorial inferioren Status zuspricht. Auf dieser Etappe seiner Reise fällt Darwin seine Urteile über das Fremde unmissverständlich aus der überlegenen Position eines Kulturträgers: Auch wenn er ein Naturforscher ist, unterliegt er dem Klischee, nämlich der Vorstellung von der kulturellen und zivilisatorischen Überlegenheit des weißen Mannes, welcher die ihm fremde Welt, die sich ihm als Natur par excellance eröffnet, zivilisieren muss. Obwohl er sich zunächst in postkolonialer Manier als Bekämpfer der kolonialen Gewaltherrschaft gibt, zeigt er sich letzten Endes als das, was er zuvor ablehnt: ein Kolonialherr, ja der Inbegriff des weißen Mannes, der die zentralen Denkfiguren des kolonialen Diskurses des 19. Jahrhunderts, zitiert', allen voran die hegemoniale Haltung zur Alterität. Formen dieser Rhetorik, d.h. der auf Dichotomien aufbauenden Evozierung der Fremden als Wilde bzw. unterentwickelte Barbaren und des Kolonialherrn als Aufklärer sind in der europäischen Literatur reichlich zu finden.

19 J. Conrad, Das Herz der Finsternis, Deutsche Übersetzung von E. Wolfgang. Berlin 2005 [1926], S. 44. 
Allerdings darf hier nicht außer Acht gelassen werden, dass zu dem Figurenensemble ja auch die Feuerländer gehören - das Auffallende dabei ist aber, dass die einzigen Indigenen, denen etwas genauere Aufmerksamkeit im Erzählprozess geschenkt wird, drei Feuerland-Indianer sind, welche in die Gefangenschaft des Kapitäns Fitz-Roy geraten und zu seinen Sklaven werden. Kennzeichnenderweise bekommen die von Fitz-Roy gefangenen Indianer von den Engländern Namen, die selbstverständlich englisch klingen. Das Manöver, das hier verwendet wird, ist ein altbekanntes: Es folgt eine Besitzergreifung des Fremden durch Benennung, wobei diesem Verhalten die Überzeugung zugrunde liegt, dass es nur eine kulturelle Ordnung gibt, die einzig wahr sei und welche als quasi Vermittler der Sprache - und somit der Kultur - fungiert. Die Sprache steht somit nicht nur im Dienste der Vereinahmung, sondern auch der Exklusion: Schweigende, ihre eigene Sprache sprechende oder der englischen Sprache nicht mächtige Feuerländer erweisen sich nämlich als stumme Objekte des kolonialen Diskurses. Am deutlichsten zeigt sich dies wohl in der folgenden Textpassage:

Obwohl es noch sehr früh am Morgen war, standen am Ufer unbeweglich ein paar dunkelhäutige Männer und Frauen, die zu dem abfahrenden Schiff hinüberschauten. Kein Laut, kein Gebärde verriet ihre Empfindung. Etwas abwärts zogen mehrere Gruppen halbnackter Neger mit einem eigentümlich modulierenden, klagenden Gesang zur Waldarbeit. Die meisten trugen primitive Werkzeuge und kleine Bündel; kraushaarige Frauen hatten ihre Kinder aufgehuckt. Peitschenknallende Aufseher zu Pferde begleiteten den Zug (S. 66).

Die von Spivak aufgeworfene Frage: Can the Subaltern speak? weist in diesem Kontext eine auffallende Aktualität auf: Als der Inbegriff der (sprachlosen) Natur können die Feuerland-Indianer keine individuelle Stimme bekommen und werden fast gänzlich aus dem werkimmanten Diskurs über das Eigene und das Fremde ausgeschlossen. Somit wird das Fremde in einer typisch kolonialen Manier nicht nur naturalisiert, sondern auch typisiert bzw. essentialisiert: zu einer gleichartig beschaffenen, seltsam anmutenden Masse, die den Europäer nur noch befremden bzw. sein Mitleid erregen kann. Allerdings gibt es in diesem Kontext eine Ausnahme (welche jedoch wohl die Regel bestätigt): Es handelt sich nämlich um Jemmy Button, einen von den drei Indianern, welcher aus der englischen Gefangenschaft in seine alte Heimat zurückkehren durfte. Als ,zivilisierter Feuerlandindianer“ (S. 60) und „ein Schiffbrüchiger der Zivilisation“ (S. 168) bekommt Jemmy eine individuelle Stimme verliehen - im gebrochenen Englisch drückt er seine Freude über die Rückkehr nach Hause aus und konversiert auf dem Schiff „Beagle“ mit Darwin und Kapitän Fitz-Roy. Dabei kann man sich aber nicht des Eindrucks erwehren, dass Jemmy nur deswegen sprechen kann, weil er fast zum Europäer wurde. Das Anliegen der Engländer, aus Jemmy, einem homme naturel, einen homme civil zu machen, erweist sich als ein typischer Mechanismus der Kolonisierung, dessen Ziel es ist, wie es Spivak in Bezug auf das Kolonialerbe bemerkte, Subjekte zu produzieren, für die der westliche Humanismus einen universellen Standard der Aufklärung darstellte. ${ }^{20}$

20 Vgl. M. Varela do Mar Castro, N. Dhawan, op. cit., S. 179. 
Neben der Ausschließung aus dem werkimmanenten Diskurs werden den indigenen Völkern entwürdigende Bilder aufgezwungen. Dies sieht man z.B. in einer Episode, in der sich der Indianer Jemmy, der einzige von den drei Feuerländern, der nach seiner Rückkehr nach Hause die europäische Identität nicht völlig abgestreift hat, sich vor seiner Familie und seinem Stamm versteckend heimlich wäscht, um einen guten Eindruck auf die vorbeifahrenden Engländer zu machen und nicht als ein verwilderter Indianer, sondern ein englischer Gentleman, wahrgenommen zu werden. Man könnte dabei den Eindruck gewinnen, dass er die an ihn herangetragenen Fremdbilder akzeptiert hat und sich fremdbestimmen ließ. Würde man diesem Gedankengang folgen, so könnte man ihn als eine weitere Exemplifizierung der in dem kolonialen Diskurs weitverbreiteten Figur des 'edlen Wilden' betrachten, dessen wohl bekanntestes Beispiel Robinson Crusoes Freitag ist. Jedoch Jemmys aufgezwungene europäische Identität mutet beinahe wie eine Nervenkrankheit an, ein obsessiver Drang, den an ihn gestellten Forderungen unbedingt gerecht zu werden:

Am nächsten Morgen sichtete Jemmy Button, der zivilisierte Feuerlandindianer, noch vor dem Posten im Ausguck die ersten Ausläufer des Festlandes. Jubelnd deutete er nach vorn und begab sich dann in die Offiziersmesse, um sich vor dem großen Spiegel für die Landung zu »schmücken». Seit seiner Verwandlung in einen „Kulturmenschen“ achtete er peinlich auf den Sitz seiner Krawatte, auf weiße Handschuhe und hochglänzende Stiefel (S. 60).

Doch wie Darwin bemerkt, seien die Bemühungen, den Indianer zu einem zivilisierten Menschen zu machen, gescheitert und seine Zwangsakkulturation sei ein Fehltritt gewesen, welcher ihn seiner „Unschuld“ (S. 174) beraubt habe. Damit präfiguriert der Held wieder einmal das Fremde als das Ursprünglich-Natürliche, das zu einer nicht wegzudenkenden Spolie seines eurozentrischen und dichotomen Denkens wird.

In der Novelle Mister Darwin macht eine Entdeckung wird das Fremde mit Hilfe von zuordnenden und hierarchisierenden Kategorien konstruiert. Die Protagonisten bleiben dem dichotomen Denken verhaftet, wobei am deutlichsten das Oppositionspaar Kultur (das Eigene) versus Natur (das Fremde) zum Ausdruck kommt. Die Kultur steht in der Novelle ausschließlich auf Seiten der Europäer: Sie wird gleichgesetzt mit der wortwörtlichen und symbolischen Gewalt gegenüber den Fremden, die nicht ihre eigene Identität behalten, geschweige denn pflegen können, sondern fremde Verhaltensmuster und Normen - allen voran die Sprache und den christlichen Glauben - aufgezwängt bekommen. Die Welt des Fremden (der Natur) muss sich dem System der Oppression fügen, welches von den Europäern ohne Rücksicht auf Verluste aufgebaut wird. Auch wenn sich vor allem im Falle Darwins bestimmte Ambivalenzen feststellen lassen und die ironisierenden Kommentare des Erzählers das Gewicht des kolonialen Diskurses etwas abschwächen, so gewinnt man im Großen und Ganzen den Eindruck, dass die Konstruktion des Fremden in der Novelle auf diskursiven Praktiken beruht, die dem kolonialen Diskurs eigen sind, allen voran der Naturalisierung und der Essentialisierung.

Wie bereits bemerkt wurde, hat eine solche Konstruktion des Fremden auch eine ideologische Funktion zu erfüllen: Es handelt sich aber nicht um jene, die 
in den Text von dem ostdeutschen Verlag eingeschrieben wurde, bzw. vom Autor intendiert war. ${ }^{21}$ Die intendierte Wirkung des Textes, dem Leser die britische Vereinnahmung des Fremden als eine von dem , besseren Deutschland' zu verdammende Praxis des britischen Imperialismus vorzuführen ${ }^{22}$, verfehlt nämlich ihr Ziel, denn eine ähnliche Vereinahmung des Fremden erfolgt seitens einer anderen, die Tarnkappe des Humanismus aufsetzenden Ideologie. Mit anderen Worten: Der Text demaskiert zwar die ,imperialistische' Politik der Vereinahmung und schreibt sich damit in das DDR-Paradigma des Antikolonialismus ein, zugleich aber betreibt der Text jene Vereinahmung selbst, nur dass er dies im Dienste eines anderen Kontrollapparats tut. In einem weiterreichenden Sinne konstituiert der Text schließlich auch eine Poetik der Kolonisierung des Lesers, welcher eine fertige Vision der Welt aufgetischt bekommen soll, die die einzig gültige Perspektive ihrer vermeintlichen Herren ist.

\section{Literatur}

\section{Primärliteratur}

Hilscher E., Mister Darwin macht eine Entdeckung, Berlin 1989.

Sekundärliteratur

(ADN), DDR verurteilt Kolonialismus und Rassenpolitik, „Neues Deutschland“, 29.01.1965, S. 7.

Cenzura w Niemczech w XX wieku. Studia, analizy, dokumenty, wybór i opracowanie Cz. Karolak, Poznań 2000.

Conrad J., Das Herz der Finsternis, Deutsche Übersetzung von E. Wolfgang. Berlin 2005 [1926].

Hawe (H. Wessel), Weltreise zur Erkenntnis, „Neues Deutschland“, 21.04.1962, S. 8. Kerner I., Postkoloniale Theorien zur Einführung, Hamburg 2012.

Lubrich O., Welche Rolle spielt der literarische Text im postkolonialen Diskurs? [in:] Archiv für das Studium der neueren Sprachen und Literaturen. 242. Band, 157, Hrsg. H. Brunner, C. Jansohn, M. Lentzen, D. Mehl, 2005, S. 16-39.

Meissner W., Eberhard Hilscher: Mister Darwin macht eine Entdeckung, ,Neue deutsche Hefte“, Berlin (West) 1981, Nr. 170, Heft 2, S. 380-381.

Neumann B., Methoden postkolonialer Literaturkritik und anderer ideologiekritischer Ansätze, [in:] Methoden der literatur- und kulturwissenschaftlichen Textanalyse, Hrsg. V. Nünning, A. Nünning, Stuttgart-Weimar 2010, S. 271-292.

21 Daher liegt es nahe, in einem weiteren Schritt die publizierte Version der Novelle mit der ursprünglichen Fassung des Textes zu vergleichen.

${ }^{22}$ An dieser Stelle sei nur Folgendes angemerkt: als Eberhard Hilscher in einem Radiogespräch in einem westdeutschen Rundfunk die Art und Weise kritisierte, wie die DDR ihre Leser zu behandeln pflegt (sie würden laut ihm eine fertige Vision der Welt serviert bekommen), wurde ihm anschließend mit „Konsequenzen“ seitens seines Verlages gedroht. Vgl. N. Nowara-Matusik..., S. 76. 
Nowara-Matusik N., Oblicza artystów. W kręgu narracji Eberharda Hilschera, Kraków 2016.

(NZ/ADN), Schluß mit dem Kolonialismus!, „Neue Zeit“", Nr. 8, April 1955, S. 2.

Sengül C., Wissenschaft als Abenteuer in Eberhard Hilschers Darwin-Erzählung [in:] Eberhard Hilscher (1927-2005). Schriftsteller und Forscher der deutschen Literatur. Pisarz i badacz literatury niemieckiej, Hrsg. G.B. Szewczyk, Świebodzin-Katowice 2010, S. 34-42.

Varela do Mar Castro M., Dhawan N., Postkoloniale Theorie. Eine kritische Einführung, Bielefeld 2005.

Walther K. Biographie in Klein- und Großformat. „Neue Deutsche Literatur“ 1963, Heft 12, S. 159-160. 